\title{
Microwave-Induced Temperature Fields in Cylindrical Samples of Graphite Powder - Experimental and Modeling Studies
}

\author{
Ethan M. Moon ${ }^{1}$, Chuqiao Yang ${ }^{1,2}$, Vadim V. Yakovlev ${ }^{1 *}$ \\ ${ }^{1}$ Department of Mathematical Sciences, Worcester Polytechnic Institute, Worcester, MA 01602, USA \\ ${ }^{2}$ Department of Engineering Sciences and Applied Mathematics, Northwestern University, Evanston, \\ IL 60208, USA
}

\begin{abstract}
ABSRACT
Microwave heating of graphite powder is the key process of a number of promising applications and emerging technologies ranging from kiln crucibles to synthesis of graphene. However, this process is typically studied in terms of structural parameters of the resulting products, whereas the prime electromagnetic and associated temperature characteristics remain insufficiently studied. In this paper, we investigate microwave heating of graphite powder in a batch (non-resonant) and waveguide (resonant) reactors using multiphysics (electromagnetic-thermal) modeling and experimentation. Temperature-dependent material parameters of graphite power are determined from measurements and appropriate physical models. Non-uniform temperature fields and their trend for relatively quick homogenization are shown to be conditioned by high values of the loss factor and thermal conductivity. These parameters are also found to be responsible for the effect of postmicrowave heating. It is demonstrated that processing of graphite power in a resonant reactor may be convenient for controlling the heating rate and the level of temperature uniformity.
\end{abstract}

Keywords: Batch and waveguide reactors, graphite, complex permittivity, microwave heating, thermal conductivity, temperature

\section{Introduction}

As a form of pure carbon, graphite appears to be a well-studied material, but recently, its behavior under microwave irradiation has received special consideration in different technical areas. The growing interest in this topic seems to be linked with the fact that microwave heating of graphite can be found in the core of a number of emerging methods of efficient production of graphene and other carbon-based materials.

- Corresponding author. Tel: +1 508831 5495; fax: +1 5088315824 .

E-mail addresses: emmoon@wpi.edu (E.M. Moon), chuqiaoyang2013@u.northwestern.edu (C. Yang), vadim@wpi.edu (V.V. Yakovlev). 
The extraordinary electrical, mechanical, optical, and thermal properties of graphene have shown that this material has the potential to revolutionize many applications, including solar cells, lithium-ion batteries, water desalination, nanocomposites, and medical diagnostics/sensors, to name a few [1]-[2]. A bottleneck for widespread adoption of graphene-based technologies is efficient mass production of graphene sheets, as the known methods are still technically demanding, expensive, and not always successful in producing graphene of desirable quality [1]-[3]. Chemistry currently plays an increasingly important role in the development of practical techniques of graphene production [3]-[5], and there are a number of chemical methods for generating graphene from graphite and its derivatives, including techniques based on exfoliation of graphite oxide [4]-[5], which appear to be scalable and afford the possibility of high-volume production. Since the exfoliation process is energy consuming and must be carried out at high temperatures, there are reports (e.g., [6]-[18]) about using microwaves as an alternative, greener and more efficient, energy source in producing graphene.

On the other hand, known as a strong absorber of microwaves, graphite has been also studied as a material that can be transformed by microwave heating, giving rise to new materials with tailored properties [19]-[20]. Other works were focused on graphite as a material for a crucible used in kilns for hybrid microwave heating [21], a substance assisting in microwave pyrolysis [22]-[23] and in microwave carbothermal reactions [24].

The quoted papers show many examples of quite peculiar and hardly predictable behavior of graphite-based samples (including production of sparks or electric arc formation [20], [25]) under microwave irradiation, but do not outline a clear picture of microwave heating of graphite as a multiphysics phenomenon. One of the reasons for that is the deficiency of systematic studies of interaction of the material with microwaves in closed cavities. In most papers dealing with microwave heating of graphite powder, the analysis was focused on structural parameters of the resulting products rather than on the prime electromagnetic and associated temperature characteristics.

In particular, to the best of the authors' knowledge, there are no studies focused on the process of formation and evolution of microwave-induced temperature fields in graphite powder. For instance, in [21], temperature of a sample heated in a microwave oven was recorded (using a thermocouple) at a single (non-specified) point, but no underlying electromagnetic characteristics of the microwave system were considered. In [24]-[25], a sample of graphite powder was heated in a dominant-mode waveguide applicator in positions corresponding to maxima of the electric and magnetic fields of an empty waveguide without studying what actually happens with the fields in the presence of the heated material. In all these papers, intrinsic non-uniformity of microwave heating was not addressed, as the considered graphite samples were chosen to be very small and uniformity of heat release there was assumed to take place. Results of computational studies of microwave heating processes involving graphite samples [23], 
[27] cannot be taken with confidence since the models employed in these works used an inadequate assumption that graphite's loss factor is negligible - in fact, graphite is known to be a strong microwave absorber (see, e.g., [20]-[21], [28]).

Overall, the lack of studies of the basics of heating of graphite by microwaves results in lack of clarity about the related physical phenomena and available options for controlling the characteristics of microwave-assisted processes involving graphite and its derivatives. Yet, understanding the features of formation of temperature fields in graphite samples may be helpful in explaining particular effects, developing applied technologies employing controllable microwave heating of graphite-based materials, and designing enlarged microwave systems for up-scaled processes.

In this paper, we report the first results in the original study of microwave-induced temperature fields in cylindrical samples of graphite powder. Temperature fields are considered in two distinct (from a microwave engineering viewpoint) systems - in a waveguide/traveling-wave reactor and in a small nonresonant batch reactors, fed by a solid-state generator allowing for rigorously controlling frequency and magnitude of the source. Temperature is measured with the use of a fiber optic sensor (in different points inside the sample) and an IR camera (on the surface of the sample). The transient heating processes in both systems are analyzed with the use of a multiphysics model computing 3D temperature fields from 3D patterns of dissipated electromagnetic power; the computation is done in the framework of an iterative procedure solving the coupled electromagnetic and heat transfer problems. Whenever possible, the model uses temperature-dependent material parameters of graphite powder that are either determined experimentally, or calculated from appropriate physical models. Computations and experiments show high heating rates and inhomogeneous distributions of dissipated microwave power that tends to even up relatively quickly. An effect of post-microwave heating is demonstrated and can be explained by the nonuniformity of microwave heating and high thermal conductivity of graphite powder. Controlling the heating rate and temperature distribution is shown to be possible in a traveling-wave system where the sliding shorting wall controls a regime of field propagation.

\section{Experimental system}

Control of microwave heating is one of the major challenges of microwave power engineering, and a search for some correlation between the temperature field and electromagnetic characteristics of the system is a crucial part of development of any efficient microwave-assisted application. Such a search, however, may result in a somewhat indefinite output due to the typical radiation properties of a magnetron, a core device of all conventional sources of microwave energy, emitting signals of a rather chaotic magnitude in a certain frequency band; examples of measured magnetron spectra can be found, 
e.g., in [29]-[31]. In order to overcome this issue, in this study, we worked with the MiniFlow 200SS (SAIREM SAS, Neyron, France), a microwave system that employs a solid-state generator providing precise control over the frequency of excitation (from 2.43 to $2.47 \mathrm{GHz}$ with a step size of $0.1 \mathrm{MHz}$ ) and the level of microwave power (from 0 to $200 \mathrm{~W}$ with a step size of $1 \mathrm{~W}$ ).

The experiments were carried out in both reactors of the MiniFlow set. The traveling-wave reactor (Fig. 1 (a), (d)) is built on a bended WR430 waveguide section with an enlarged cylindrical space for the sample to be processed in the system. This space is bounded by a cylindrical Teflon tube (internal diameter $60 \mathrm{~mm}$, height $94 \mathrm{~mm}$ ) and accessible (for measurements and visual observation) through two (vertical and horizontal) metal tubes serving, due to their lengths and diameters, as cutoff circular waveguides preventing leakage from the cavity. The reactor is excited through a coax-to-waveguide transition. On the opposite end, the terminal section contains a sliding shorting wall controlling wave propagation in the system.

The batch reactor (Fig. 1 (b)) consists of a small cylindrical cavity (internal diameter $63 \mathrm{~mm}$, height $46 \mathrm{~mm}$ ) containing a concentrically positioned cylindrical Teflon cup (internal diameter $23 \mathrm{~mm}$, height 67 $\mathrm{mm}$ ) intended for holding the sample in a cylindrical vessel which is put into the system through a cylindrical hole in the cavity's upper lid. Similarly, due to the large thickness of the lid (53 mm), this hole serves as a cutoff waveguide. The cavity is fed via a coaxial cable whose internal conductor is connected with a metal T-shaped structure inside the cavity.

In the experiments, we worked with synthetic graphite powder (Sigma-Aldrich Co., LLC) whose particles had diameter $<20 \mu \mathrm{m}$ in a Pyrex vial (internal diameter $20 \mathrm{~mm}$, height $75 \mathrm{~mm}$ ). Internal temperature of the sample was measured by a fiber optic sensor (Neoptix, Inc.) held, except its tip, inside a glass capillary (ID $2 \mathrm{~mm}$ ) and placed at different heights $h$ in the cylindrical sample in the vial (as shown in Fig. 1 (c)). Temperature on the cylindrical surface of the sample was also recorded through the side hole (diameter $15 \mathrm{~mm}$ ) of the waveguide reactor (Fig. 4 (a), (d)) with the use of the FLIR i7 infrared camera (FLIR Systems, Inc.) capable of monitoring the temperature field against the associated scale which is interpreted as the available minimum and maximum temperature within the pictured field.

\section{Computational technique}

Computer simulation of electromagnetic and thermal processes in both MiniFlow reactors was carried out in accordance with the computational scheme for an iterative solution of the electromagnetic-thermal two-way coupled problem [32]-[34]. Aiming to reach a high level of adequacy in representing microwave heating scenarios, this advanced technique accounts for temperature-dependent electromagnetic and thermal material parameters of the heated material and upgrades their values in all relevant grid cells after 


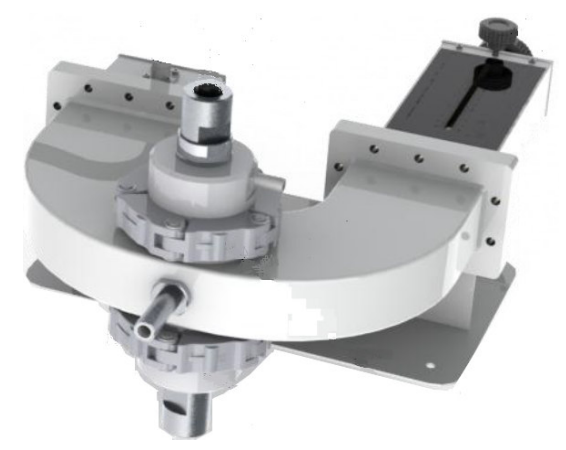

(a)

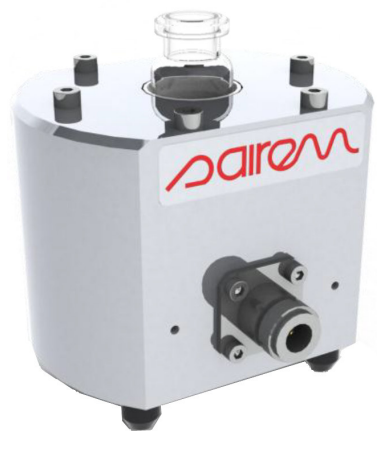

(b)

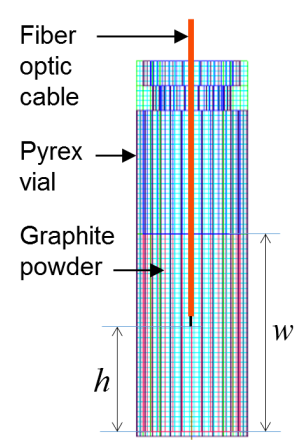

(c)

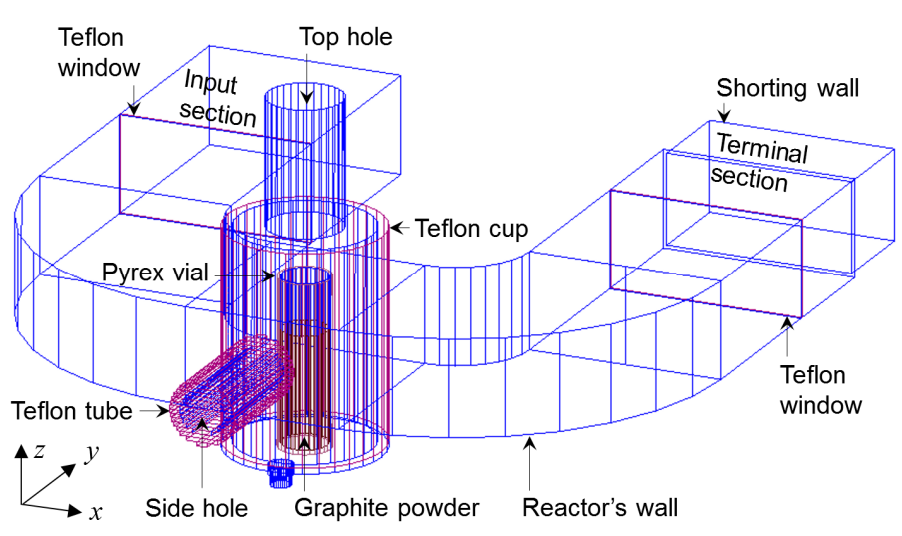

(d)

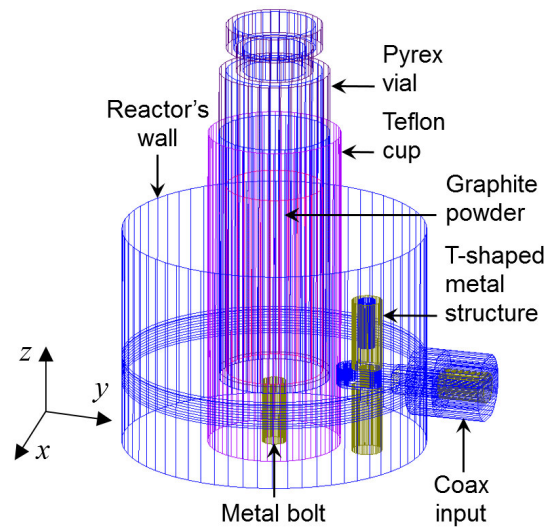

(e)

Fig. 1. MiniFlow 200SS waveguide (a) and batch (b) reactors and 3D views of their internal structure (d) and (e), respectively; position of a fiber optic sensor measuring internal temperature of the sample (c).

each heating time step. Internal structure of the reactors was precisely reproduced in parameterized 3D models developed for the full-wave 3D conformal finite-difference time-domain (FDTD) simulator QuickWave ver. 2013 [35]. The internal layouts of the reactor's components in the models are shown in Figs. 1 (d) and (e). The computational algorithm was implemented using the core electromagnetic solver of the QuickWave package and its dedicated procedure for solving the heat transfer problems - the QuickWave Basic Heating Module.

The boundary conditions for the electromagnetic portion of the problem were applied assuming that the internal surfaces of the reactors are made of perfect metal. The condition of thermal insulation (Neumann boundary condition) was applied on the external surface of the Teflon cup (containing the Pyrex vial with graphite powder in direct contact) in the batch reactor and on the external surface of the Pyrex vial (separated from the Teflon cup by an air gap) in the waveguide reactor. The convective (Robin) boundary condition was applied on the interface between graphite and Pyrex, with the convective heat transfer coefficient, determined by a series of computational experiments, equal to $0.05 \mathrm{Wcm}^{-2} \mathrm{~K}^{-1}$. 
Simulations were run on a Dell T-4700 workstation (64-bit Windows XP) with 16 GB RAM and two quad-core Intel Xeon 3.20 GHz processors. To ensure high accuracy of the solution of the prime electromagnetic problem and minimize CPU time, the models representing both MiniFlow reactors were discretized with non-uniform meshes with cell sizes adjusted in accordance with the wavelengths in the media. For both reactors, the models consisted of 1.4 to 1.7 million cells (depending on the height of the graphite sample). When solving the coupled problem, the heating time step in the iterative process was set, depending on the heating rate, at 0.5 to $3 \mathrm{~s}$, and computation of temperature evolution in the entire transient process took 40 to $120 \mathrm{~min}$ of CPU time.

\section{Material parameters of graphite powder}

While precise reproduction of the entire geometry of a microwave system and accuracy of the applied numerical technique can be considered necessary conditions for sensible computer simulations of microwave heating, for reasonably adequate computational studies of a particular process, the developed model should be supplied with reliable input data on temperature-dependent media parameters of all the materials involved [34], [36]. Therefore, to mimic microwave heating of graphite powder in the MiniFlow reactors, the electromagnetic and thermal properties (i.e., dielectric constant $\varepsilon^{\prime}$, the loss factor $\varepsilon^{\prime \prime}$, specific heat $C_{p}$, density $\rho$, and thermal conductivity $k$ ) of Teflon, Pyrex and graphite powder should be specified in the models in the considered temperature range. For graphite powder, there is no consensus on such a data set in literature; this section shows how these parameters were chosen for simulations described in this paper.

\subsection{Dielectric properties}

Complex permittivity $\left(\varepsilon=\varepsilon^{\prime}-j \varepsilon^{\prime \prime}\right)$ of graphite powder used in the experiments (described in Section 6) was measured at $2.45 \mathrm{GHz}$. The powder was loosely poured (i.e., not pressurized) in an open-shield coaxial cell, and $\varepsilon^{\prime}$ and $\varepsilon^{\prime \prime}$ were determined (at a room temperature) similarly to Agilent's coaxial probe method involving measurement of reference materials; the results recently reported in [37] are shown in Table 1. Comparing these values with the data obtained in [28] by a similar measurement technique, but for the powder of larger particle sizes and three different densities, one can see that for larger particles, the loss factor is lower: that can be explained by a decreased contact area between the particles, and thus degenerated conditions for electrical conductivity. Also, the values of dielectric constants are of the same order for the powders of smaller and larger particles. This is consistent with the observation that electromagnetic characteristics of a waveguide system containing a powder inclusion depend on the solids 


\section{Table 1}

Complex permittivity of graphite powder at $2.45 \mathrm{GHz}$.

\begin{tabular}{ccccc}
\hline Ref. & & {$[28]$} & & This paper [37] \\
\hline Particles sizes $(\mu \mathrm{m})$ & & 80 & & $<20$ \\
Density $\left(\mathrm{g} / \mathrm{cm}^{3}\right)$ & 0.215 & 0.249 & 0.256 & 0.276 \\
$\varepsilon^{\prime}$ & 26.0 & 23.5 & 19.1 & 21.6 \\
$\varepsilon^{\prime \prime}$ & 10.9 & 12.8 & 14.6 & 27.5 \\
\hline
\end{tabular}

volume fraction (and thus density) rather than particle sizes [38]. This suggests that the measured values of complex permittivity can be considered accurate.

In the absence of data on $\varepsilon^{\prime}$ and $\varepsilon^{\prime \prime}$ at higher temperatures, in this paper, complex permittivity of graphite powder was assumed to be temperature independent. In accordance with [39]-[40], at $2.45 \mathrm{GHz}$, such related materials as graphitized carbon blacks and expanded graphite composites have insignificant variations of $\varepsilon^{\prime}$ and $\varepsilon^{\prime \prime}$ at temperatures below $100-120^{\circ} \mathrm{C}$. For the present first round of computational studies, the assumption of temperature independence was found to be reasonable and practical.

\subsection{Specific heat}

In determining thermal material parameters via physical models, graphite powder can be considered as a mixture of graphite solid and air. For the specific heat of solid graphite, the temperature characteristic suggested in [41] and adopted as a standard reference is given by the formula:

$$
C_{P}=4.184\left(0.5421-2.4267 \cdot 10^{-6} T-90.2725 T^{-1}-4.3449 \cdot 10^{4} T^{-2}+1.5931 \cdot 10^{7} T^{-3}-1.4369 \cdot 10^{9} T^{-4}\right)
$$

where $T$ is temperature in the interval from -23 to $2727^{\circ} \mathrm{C}$. For graphite solid heated in a microwave reactor, specific heat can be seen as a ratio of the heat absorbed by the material $Q$ and temperature rise $\Delta T$ times mass $m$. Assuming that graphite powder is produced from graphite solid by adding air and that the mass of air is negligible, specific heat may be taken to be the same as that of solid graphite. The temperature characteristic of specific heat for graphite powder can thus be determined from (1); Table 2 contains a set of values of $C_{p}\left(\mathrm{Jcm}^{-1} \mathrm{C}^{-1}\right)$ calculated for temperatures in the range from 20 to $200^{\circ} \mathrm{C}$.

\subsection{Density}

The true density $\rho_{s}$ of solid graphite is known to be $2.1-2.3 \mathrm{~g} / \mathrm{cm}^{3}$ [42-43]. As graphite is a porous material, in reality its density is less: e.g., Poco Graphite, Inc. produces polycrystalline graphite in the 


\section{Table 2.}

Graphite powder: temperature characteristic of specific heat.

\begin{tabular}{lcccccccccc}
\hline$T\left({ }^{\circ} \mathrm{C}\right)$ & 20 & 40 & 60 & 80 & 100 & 120 & 140 & 160 & 180 & 200 \\
$\left.C_{p}\left(\mathrm{Jcm}^{-10} \mathrm{C}^{-1}\right)\right)$ & 0.693 & 0.750 & 0.807 & 0.864 & 0.919 & 0.972 & 1.023 & 1.072 & 1.118 & 1.162 \\
\hline
\end{tabular}

density range $1.3 \leq \rho_{s} \leq 1.9 \mathrm{~g} / \mathrm{cm}^{3}$. For graphite powder, the apparent (or bulk) density $\rho$ of the sample can be determined from the sample's volume and mass. For the samples in our experiments, $\rho=0.276$ $\mathrm{g} / \mathrm{cm}^{3}$. Apart of very high temperatures of graphitization $\left(2,000^{\circ} \mathrm{C}\right.$ and above), the apparent density does not depend on temperature, so, in this paper, $\rho$ is considered constant for the entire temperature interval from 20 to $200^{\circ} \mathrm{C}$.

\subsection{Thermal conductivity}

One of the issues in modeling microwave heating of graphite and its derivatives is anisotropic thermal conductivity. While at room temperature the values of in-plane thermal conductivity $k_{\|}$of solid highly oriented pyrolytic graphite can reach $20 \mathrm{Wcm}^{-1} \mathrm{C}^{-1}$, thermal conductivity in a perpendicular direction $k_{\perp}$ is reported to be around $0.06 \mathrm{Wcm}^{-1} \mathrm{C}^{-1}$ [44]-[45]. Anisotropy of the medium formed by micro- and nanoscale graphite particles, however, is not so well studied; it appears that, in the powder with wellmixed particles, the conditions for very high thermal conductivity may be made available for all directions. The absence of a quantitative measure of re-orientation of the platelets makes characterization of graphite powder in terms of its thermal conductivity $k$ of graphite powder a non-trivial problem. In this paper, the value of a room-temperature thermal conductivity of the experimental sample is determined from direct measurement, and temperature-dependent $k$ is found from a suitable physical model for thermal conductivity of porous materials.

As a mixture of a solid fraction and air, graphite powder may be represented by a model for the effective thermal conductivity of a medium made up from inclusions of one substance embedded in a matrix of a different material; most such models are developed for particles of different shapes (spheres, cylinders, and irregular particles) with point contacts between them [46]-[47]. In the Zehner-Schlinder model [48], the interface between the fractions is characterized by the shape factor $B$ : when $B=0$, the boundary is reduced to the $z$-axis with no solid volume; when $B=1$, the solid is a sphere; finally, when $B \rightarrow \infty$, the solid occupies the entire cylinder.

Under the assumption of the point contacts, the Zehner-Schlinder model (thoroughly validated by diverse experimental data and found fairly robust and accurate [46]) is limited to the use with mixtures for which the ratio of their components' thermal conductivities is less than $10^{3}$. This means that this model 
may produce unreliable results if the solid fraction of graphite powder is characterized by in-plane thermal conductivity - in this case, if $k_{\|} / k_{\text {air }}>10^{4}$.

Here we compute $k$ using the approach suggested by Hsu et al [46] and based on modification of the Zehner-Schlinder model for a packed-sphere bed with finite contact areas (rather than point contacts) between spheres. Finite-area contacts between spheres are likely to occur due to external loads or owing to their own weight; they also may support higher conductivity of the embedded substance. To this end, the interface between the fractions is characterized with the use of the radius of the contact area which depends on $B$ and the deformation factor $\alpha$. The modified Zehner-Schlinder model is in a good agreement with experimental data at the values of thermal conductivity ratio up to about $10^{4}$ [46].

Following Hsu et al [46], the effective thermal conductivity of the graphite particles/air mixture (i.e., graphite powder) can be found from the formula:

$k=k_{\text {air }}\left\{1-\sqrt{1-\varphi}+\frac{\sqrt{1-\varphi}}{\lambda}\left[1-\frac{1}{(1+\alpha B)^{2}}\right]+\frac{2 \sqrt{1-\varphi}}{1-\lambda B+(1-\lambda) \alpha B}\left[\frac{(1-\lambda)(1+\alpha) B}{[1-\lambda B+(1-\lambda) \alpha B]^{2}} \ln \frac{1+\alpha B}{(1+\alpha) \lambda B}-\frac{1+B+2 \alpha B}{2(1+\alpha B)^{2}}-\frac{B-1}{[1-\lambda B+(1-\lambda) \alpha B](1+\alpha B)}\right]\right\}$

where $\lambda=k_{\text {air }} / k_{s}, k_{s}$ is thermal conductivity of the solid fraction, $\varphi=\left(\rho_{s}-\rho\right) /\left(\rho_{s}-\rho_{\text {air }}\right)$ is the porosity of the mixture, and the shape factor $B$ is determined, by iterations for given values of $\alpha$ and $\varphi$, from the equation

$$
\varphi=1-\frac{B^{2}}{(1-B)^{6}(1+\alpha B)^{2}}\left[B^{2}-4 B+3+2(1+\alpha)(1+\alpha B) \ln \frac{(1+\alpha) B}{1+\alpha B}+\alpha(B-1)\left(B^{2}-2 B-1\right)\right]^{2}
$$

where the deformation factor $\alpha$ can be set to 0.002 as for this value the modified Zehner-Schlinder model was found to be matching the experimental results at high thermal conductivity ratios [46].

In accordance with (2)-(3), thermal conductivity of graphite powder depends on two parameters, $\rho_{s}$ and $k_{s}$, whose values are known to be in the intervals

$$
\begin{gathered}
1.3 \leq \rho_{s} \leq 1.9 \mathrm{~g} / \mathrm{cm}^{3}, \text { and } \\
k_{\perp}(T) \leq k_{s} \leq=k_{\|}(T) .
\end{gathered}
$$

This means that, if combined with experimental data on $k_{\perp}(T)$ and $k_{\|}(T)$ [44], equations (2)-(3) can be used for finding the bounds of $k(T)$ for any graphite powder. Corresponding characteristics (computed with $k_{\text {air }}=2.57 \times 10^{-4}\left(\mathrm{Wcm}^{-1} \mathrm{C}^{-1}\right)$ [49] $)$ are shown in Fig. 2 . It is seen that while the true density $\rho_{s}$ of 


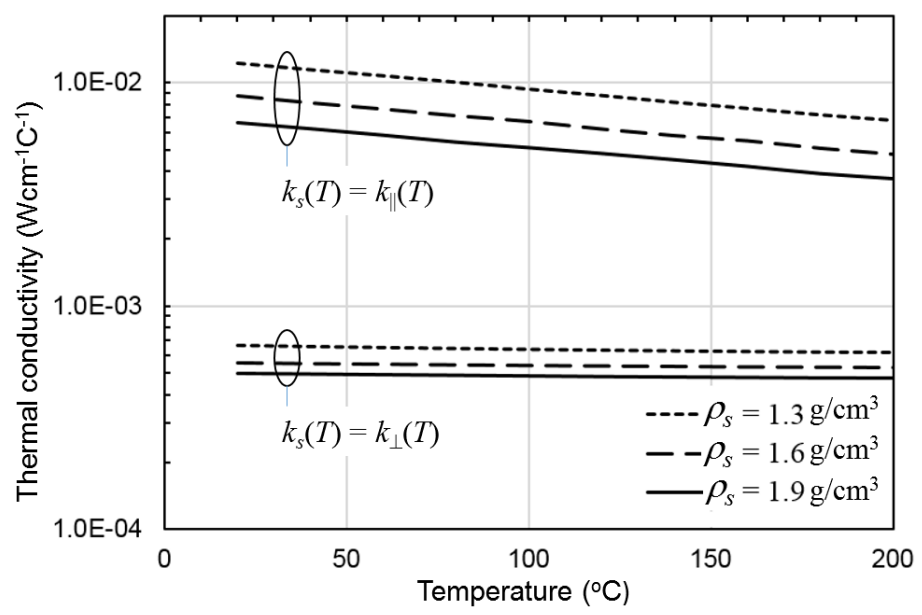

Fig. 2. Temperature characteristics of thermal conductivity of graphite powder for the extreme possible values of thermal conductivity of graphite solid $k_{s}$.

graphite solid makes a relatively modest impact on thermal conductivity of graphite powder, an uncertainty in thermal conductivity of solid fraction is critical: depending on the orientation of the graphite platelets in a given powder (in other words, on the value of $k_{s}$ between $k_{\perp}$ and $k_{\|}$) there could be up to 20 times difference in the resulting value of $k$.

Thermal conductivity of the experimental sample of graphite powder at room temperature was experimentally determined from thermal resistivity of the powder placed in a Teflon tube between two (hot and cold) brass disks; the details of the technique have been recently reported in [37]. The experimental value $k^{*}=0.0191\left(\mathrm{Wcm}^{-1} \mathrm{C}^{-1}\right)$ turned out to be closest to the value (0.0123) obtained from (2)-(3) for maximum of $k_{s}=k_{\|}$, minimum of $\rho_{s},=1.3 \mathrm{~g} / \mathrm{cm}^{3}$ and $T=20^{\circ} \mathrm{C}$. Some difference between the computed and measured values is likely influenced by the fact that the measurement was done with pressurized powder, in which $k$ is naturally expected to be higher than in the sample of loose graphite powder that was used in microwave heating experiments (Section 6). This suggests that the corresponding temperature characteristic of $k$ (shown by the dotted curve on the top of Fig. 2) can be taken as the input data in the related multiphysics modeling; the resulting function can be approximated by the linear function:

$$
k\left(\mathrm{Wcm}^{-1} \mathrm{C}^{-1}\right)=0.0127-0.00003 T
$$

\section{Results}

Here we discuss temperature (and relevant electromagnetic) characteristics of microwave heating of graphite powder in the MiniFlow waveguide and batch reactors; the observations are made both from experimentation and simulation. In the model, in accordance with standard handbook and manufacturers' 


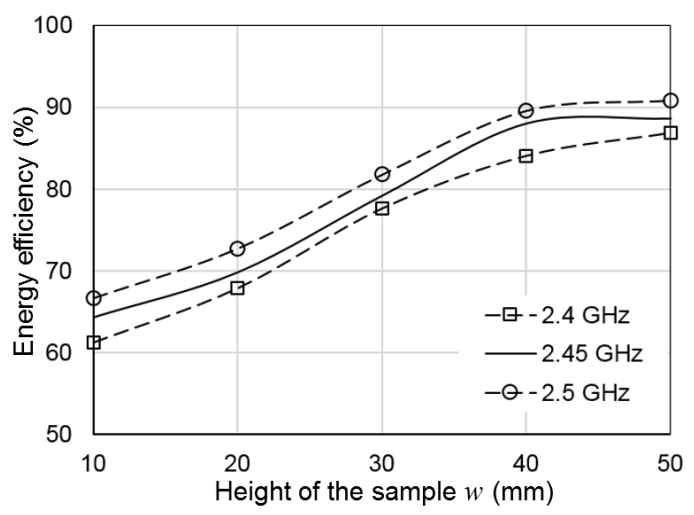

Fig. 3. Microwave heating of graphite powder in the MiniFlow 200SS batch reactor: simulated energy coupling as function of the sample size; simulated for the material parameters of the powder with $\rho_{s}=$ $1.3 \mathrm{~g} / \mathrm{cm}^{3}$ and $k_{s}(T)=k_{\|}(T)$ at the initial temperature of $20^{\circ} \mathrm{C}$.

\section{Table 3.}

Electromagnetic and thermal material parameters used in the model.

\begin{tabular}{lccccc}
\hline Parameters & $\varepsilon^{\prime}$ & $\varepsilon^{\prime \prime}$ & $C_{p}\left(\mathrm{Jg}^{-10} \mathrm{C}^{-1}\right)$ & $\rho\left(\mathrm{gcm}^{-3}\right)$ & $k\left(\mathrm{Wcm}^{-10} \mathrm{C}^{-1}\right)$ \\
\hline Pyrex & 4.6 & 0.0316 & 0.79 & 2.23 & 0.0110 \\
Teflon & 2.1 & 0.0003 & 1.30 & 2.20 & 0.0025 \\
\hline
\end{tabular}

data, all the material parameters of Teflon and Pyrex were assumed to be temperature-independent; their numerical values are given in Table 3.

Fig. 3 shows how microwave energy absorbed by the graphite sample in the batch reactor depends on the sample's height; the energy efficiency is calculated here as the derivative of the reflection coefficient computed at the input port of the system [33]-[34]. One can see that the larger the sample's volume, the more energy is absorbed. This is consistent with the fact that the batch reactor is a small, non-resonant cavity in which reflections and the electric field are conditioned by the geometry of the material and its complex permittivity.

Evolution of the microwave-induced temperature field in the batch reactor can be estimated from the patterns in Fig. 4 (a). The field appears to be slightly asymmetric towards the excitation elements of the reactor and overall is characterized by the absence of strong hot spots; the relatively spread patterns can be explained by the high thermal conductivity of graphite powder. Since in this computation $k$ is specified with an intrinsic uncertainty, as the left and right endpoints of (4a), (4b), respectively, two computational tests were also performed for alternative values of $\rho_{s}$ and $k_{s}(T)$ - for the opposite endpoints of these inequalities; the patterns produced by these computations are shown in Fig. 4 (b), (c). The results reveal the significant influence of thermal conductivity on the heating pattern and the heating rate: if graphite 

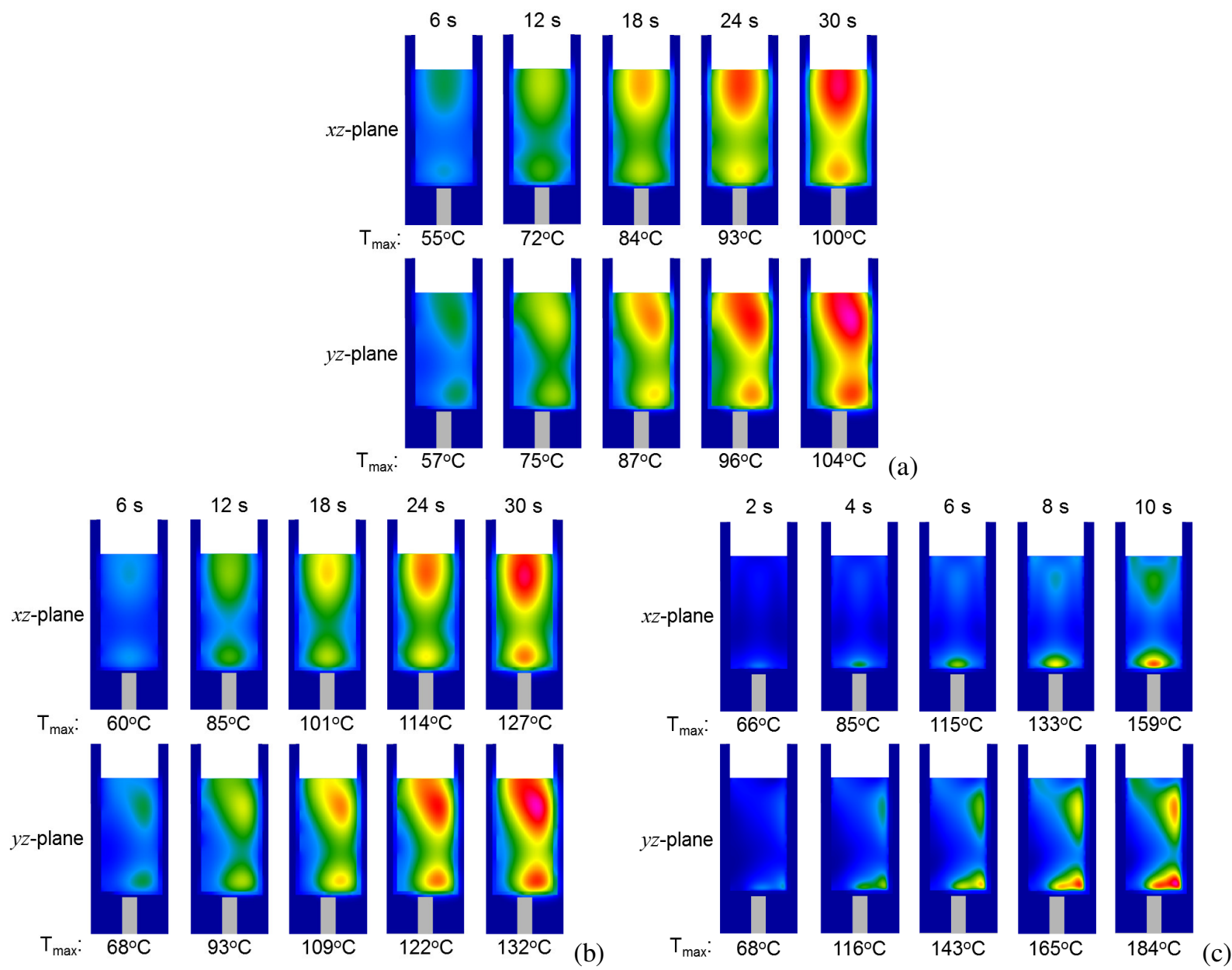

Fig. 4. Simulated temperature fields of microwave heating (initial temperature $20^{\circ} \mathrm{C}$ ) visualized in the coordinate plane through the lower part of the Teflon cup containing the Pyrex vial with graphite powder $(w=40 \mathrm{~mm})$; batch MiniFlow 200SS system with $40 \mathrm{~W}$ microwave power at $2.45 \mathrm{GHz}$ and temperaturedependent thermal conductivity corresponding to $\rho_{s}=1.3 \mathrm{~g} / \mathrm{cm}^{3}, k_{s}(T)=k_{\|}(T)(\mathrm{a}), \rho_{s}=1.9 \mathrm{~g} / \mathrm{cm}^{3}, k_{s}(T)=$ $k_{\|}(T)(\mathrm{b})$, and $\rho_{s}=1.3 \mathrm{~g} / \mathrm{cm}^{3}, k_{s}(T)=k_{\perp}(T)(\mathrm{c})$; each set of patterns is normalized to the maximum temperature of the last time instance of the heating process.

powder had a notably lower value of $k$, microwave energy would be released in strongly localized hot spots and, as the loss factor of graphite powder is very high (Table 1), it would be released very quickly.

Comparison of patterns (a)-(c) also suggests that for determination of $k$ from (2)-(3) (and for its subsequent use in multiphysics simulation) knowledge of exact value of $\rho_{s}$, in contrast to $k_{s}(T)$, is not critical: the endpoints of the interval (4a) result in somewhat different heating rate, but the heating pattern is kept almost the same. 


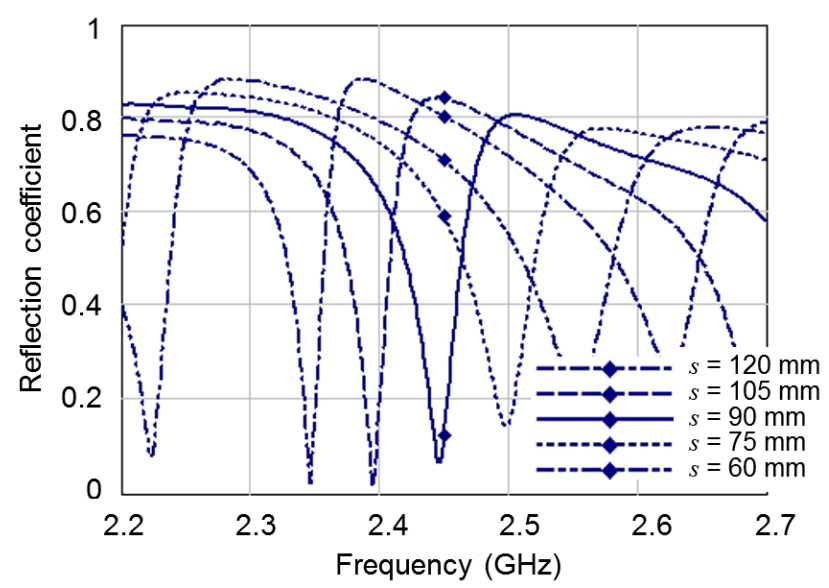

Fig. 5. Computed frequency characteristics of the reflection coefficient in the MiniFlow 200SS waveguide reactor with a cylindrical sample of graphite powder $(w=50 \mathrm{~mm})$ for different positions $s$ of the shorting wall.

Another computational test was performed in order to check whether microwave heating of graphite powder in the batch reactor depends on frequency. The dotted curves shown in Fig. 3 indicate that energy efficiency of the system does not change much in the ISM band around $2.45 \mathrm{GHz}$, so an alternation in frequency may result only in a minor change in heating rate, but not in temperature distribution. As such, the impact of this parameter is similar to the one made by the level of microwave power.

All the results presented above suggest that the means of control over microwave heating of graphite powder in a small, non-resonant batch reactor are fairly limited. In contrast to that, in the MiniFlow's waveguide reactor, both heating rate and heating patterns in the given sample of graphite powder can be conveniently controlled by one "external" parameter of the system. Our computational and experimental results show that electromagnetic and thermal characteristics of the reactor (including temperature distributions in the graphite sample) are strongly influenced by the position of the sliding shorting wall. It was found that in order to put the reflection coefficient of the system in a deep resonance (as shown in Fig. 5), the distance $s$ from this wall to the Teflon window in the flange cross-section of the terminal section (Fig. 1(a), (d)) should be around $90 \mathrm{~mm}$. Accordingly, in the waveguide section preceding the sample, a traveling wave is observed (Fig. 6(a)), most of the input microwave power is absorbed by the sample, and the graphite powder is heated quite quickly - for example, in the process whose temperature fields are shown in Fig. 7 (a), the maximum temperature of $200^{\circ} \mathrm{C}$ is reached in less than $10 \mathrm{~s}$. The temperature patterns follow the distribution of the electric field, which has a higher magnitude on the farther side of the cylindrical sample (Fig. 6 (a)). When changing $s$, the resonance is shifted away from $2.45 \mathrm{GHz}$, the reflections significantly rise, the electric field assumes the pattern of a standing wave (Fig. 


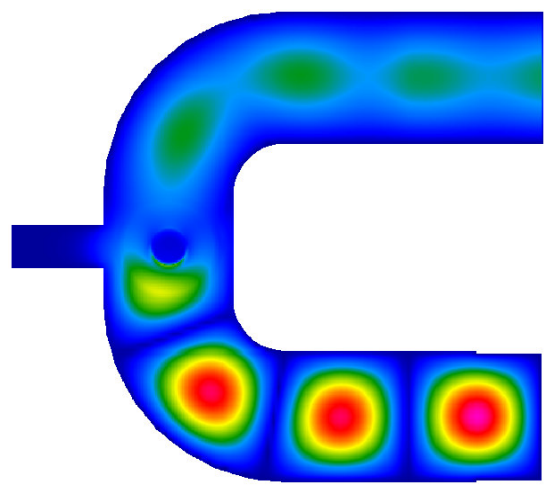

(a)

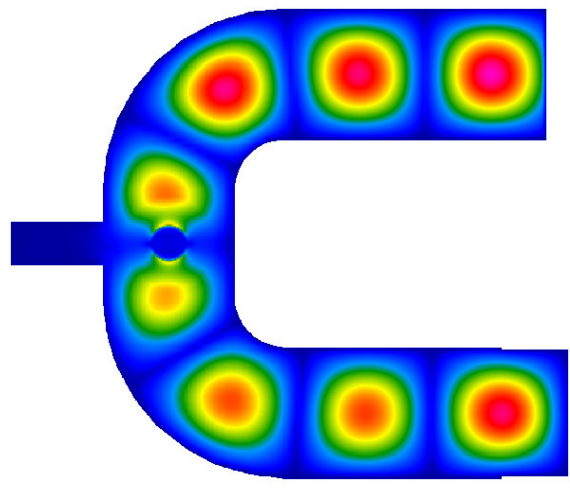

(b)

Fig. 6. Simulated time-average electric fields at $2.45 \mathrm{GHz}$ in the $x y$-coordinate plane through the center of the MiniFlow 200SS waveguide system containing the Pyrex vial with graphite powder $(w=50 \mathrm{~mm})$; shorting wall at the position of $s=90 \mathrm{~mm}$ (a) and $105 \mathrm{~mm}$ (b).

6 (b)), the sample absorbs much less microwave power, the heating rate significantly drops (e.g., in accordance with Fig. 7 (b), with $s=105 \mathrm{~mm}, 90^{\circ} \mathrm{C}$ is reached for as long as about $30 \mathrm{~s}$ ), and the temperature field becomes more spread throughout the sample (as in Fig. 7 (b)) due to the role of its high thermal conductivity.

Measurement of temperature characteristics of microwave heating of graphite powder in both MiniFlow reactors confirms a considerable non-uniformity of heating and also reveals an effect of the socalled post-microwave heating. With the use of a fiber optic sensor, temperature was measured at internal points of the samples; an infrared camera was used to visualize temperature distribution on the cylindrical surface facing the side hole of the waveguide reactor. The effect is illustrated here by experimental data obtained from three different heating scenarios.

Figs. 8-9 show the time-temperature characteristics at the points located at different heights $h$ inside the sample in the batch and waveguide reactors, respectively. All the curves indicate that temperatures at the measured points continue to grow after shutting down the microwave generator - in our experiments, for 2 to $60 \mathrm{~s}$ depending on the level of microwave power, duration of microwave heating, and location of the point. It is also seen from Fig. 10 that the duration of post-microwave heating depends on the rate of microwave heating; the latter can be controlled, as mentioned above, by the position $s$ of the shorting wall in the waveguide reactor. The effect can be explained by the strong non-uniformity of microwave heating at a high rate and a significant subsequent heat diffusion from overheated areas to the underheated ones, due to the high thermal conductivity of graphite powder.

This interpretation of post-microwave heating as of thermal relaxation is well consistent with the time evolution of temperature fields revealed by the simulation (Figs. 4 and 7), but it does not fully explain the 

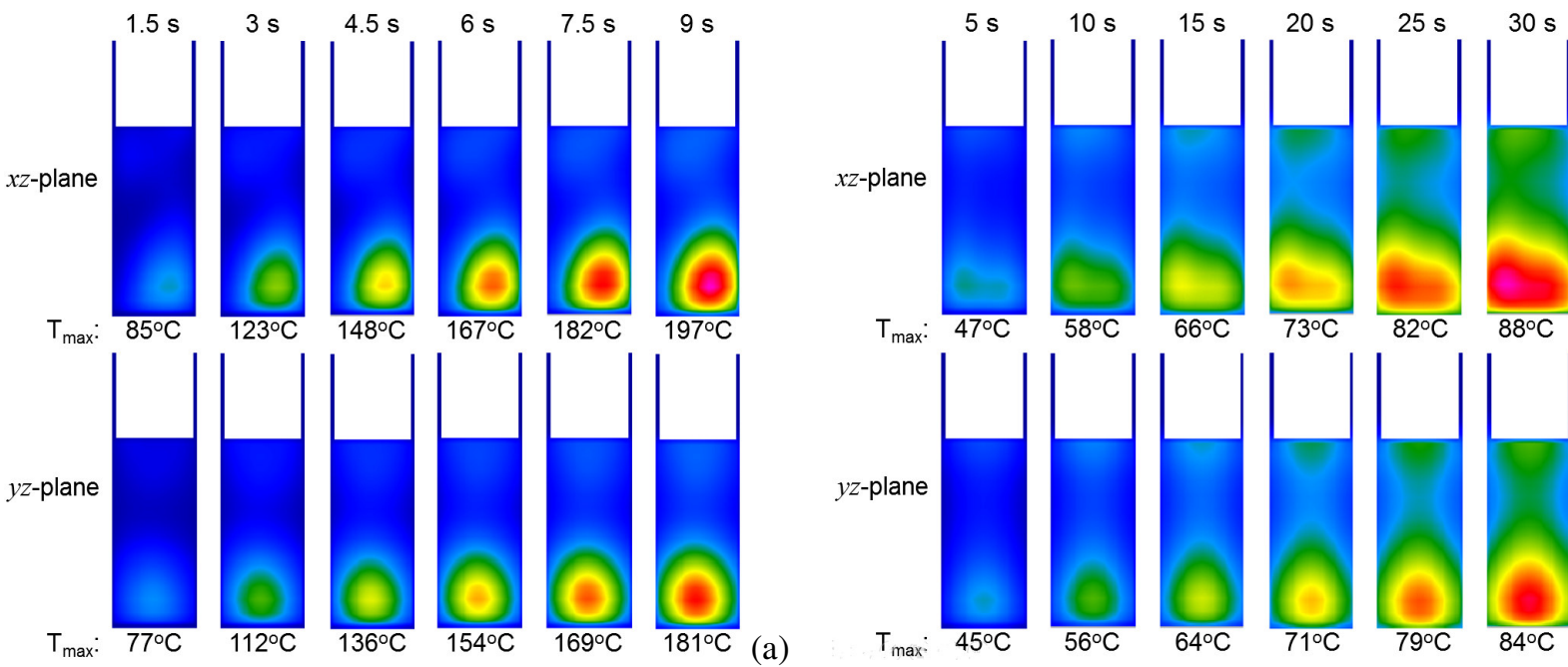

(b)

Fig. 7. Simulated temperature fields of microwave heating (initial temperature $20 \mathrm{C}$ ) in the coordinate plane through the lower part of the Pyrex vial with graphite powder $(w=50 \mathrm{~mm})$; MiniFlow 200SS waveguide reactor with traveling $(s=90 \mathrm{~mm})$ (a) and standing $(s=105 \mathrm{~mm})(\mathrm{b})$ waves and $100 \mathrm{~W}$ microwave power at $2.45 \mathrm{GHz}$; temperature-dependent thermal conductivity corresponding to $\rho_{s}=1.3$ $\mathrm{g} / \mathrm{cm}^{3}$ and $k_{s}(T)=k_{\|}(T)$; each set of patterns is normalized to the maximum temperature of the last time instance of the heating process.

shapes of all measured characteristics: it is seen that the rate of post-microwave heating may be higher at the points of high microwave-induced temperatures. We believe that in these cases additional heat is generated by better access of near-surface particles to oxygen. Indeed, volatile reaction products of carbon monoxide and carbon dioxide are known to be formed at all temperatures above $25^{\circ} \mathrm{C}$ [50], and they are thus likely to form in the course of microwave heating of graphite powder, especially, in the upper part of the cylindrical sample, as illustrated by the top curves in Fig. 9 (a), (b).

For the scenario with the $50 \mathrm{~mm}$ sample in the waveguide reactor, the reflected power was both measured and simulated; the resulting ranges are highlighted in Fig. 9 (b). Given the intrinsic uncertainty in characterization of material parameters of graphite powder in the model, the presented level of closeness of the experimental and modeling results can be considered satisfactory. Since the same electromagnetic model (running merely with an alternative (sinusoidal rather than pulse) excitation) was involved in the multiphysics simulations, one can suggest that the reported above computational results on the evolution of microwave-induced temperature fields may quite adequately represent the actual thermal characteristics of microwave heating of graphite powder in the considered batch and waveguide reactors. This suggestion is directly supported by a full consistency of the IR thermal images in Fig. 10 (showing the transient temperature distribution on the surface of the central section of the sample in the 


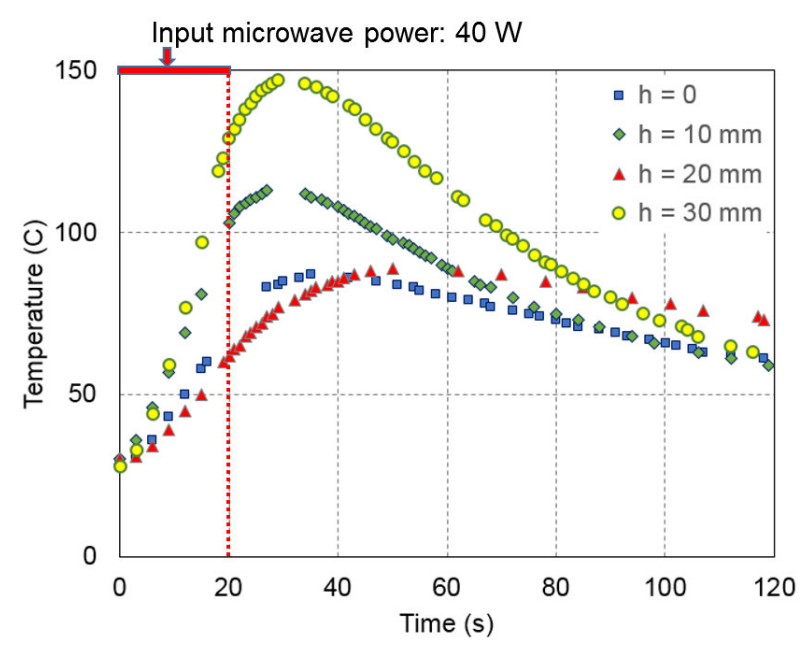

(a)

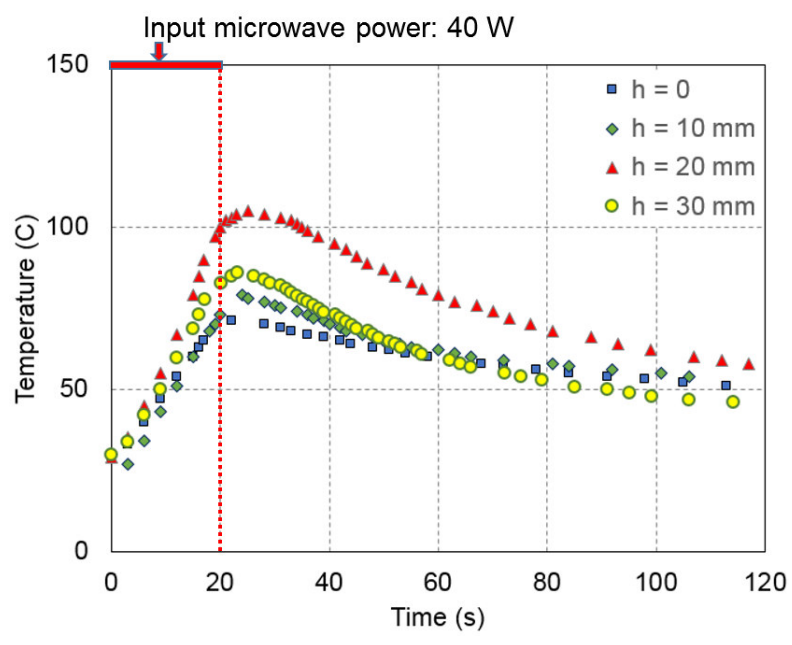

(b)

Fig. 8. Temperature evolution in different positions $h$ along the axis (a) and near the wall (b) of the cylindrical sample (diameter $20 \mathrm{~mm}$, height $38 \mathrm{~mm}$ ) of graphite powder in the Pyrex vial (outer diameter $23 \mathrm{~mm}$, height $74 \mathrm{~mm}$ ); MiniFlow 200SS batch reactor; incident microwave power (40 W, $2.45 \mathrm{GHz}$ ) is applied for $20 \mathrm{~s}$.

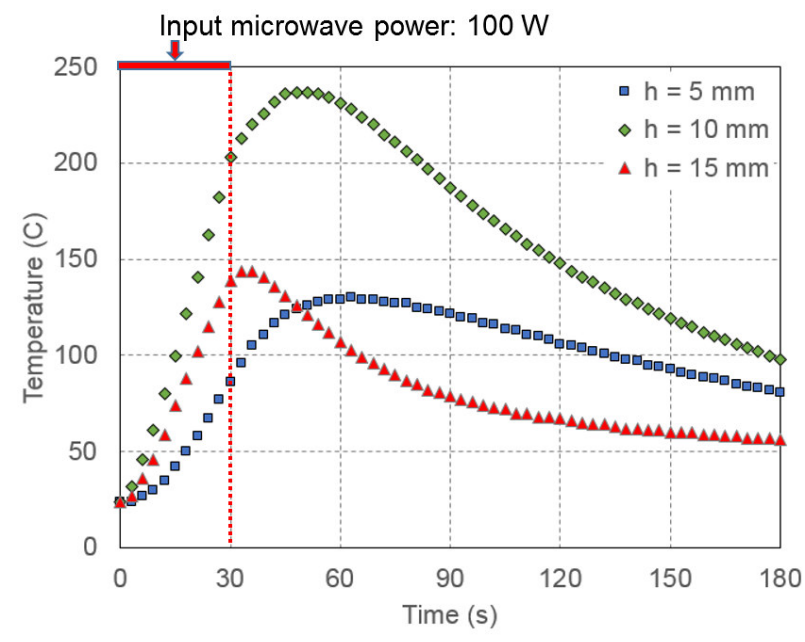

(a)

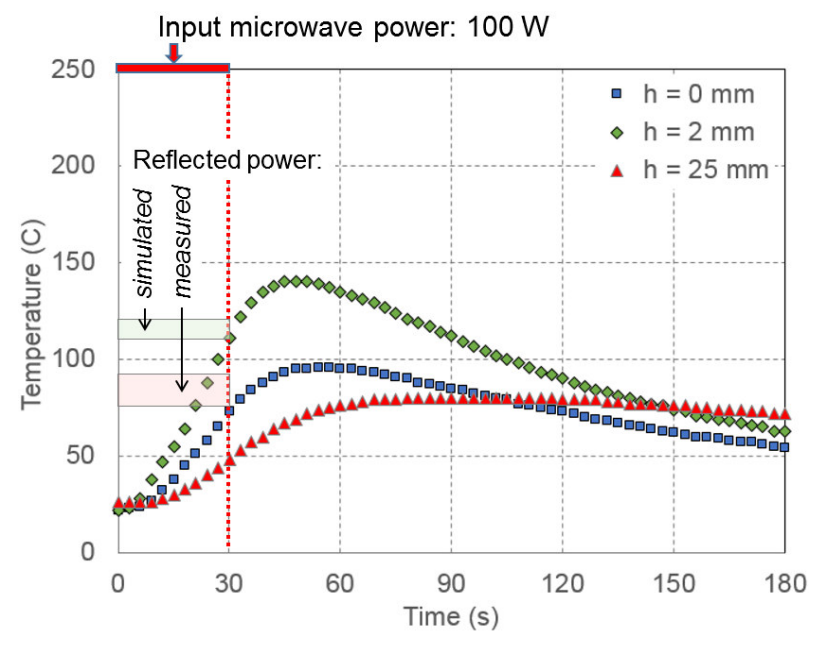

(b)

Fig. 9. Temperature evolution in different positions $h$ along the axis of the cylindrical sample (diameter $20 \mathrm{~mm}$, height $20 \mathrm{~mm}$ (a) and $50 \mathrm{~mm}$ (b)) of graphite powder in the Pyrex vial (outer diameter $23 \mathrm{~mm}$, height $74 \mathrm{~mm}$ ); MiniFlow 200SS traveling-wave reactor with $s=104 \mathrm{~mm}$; incident microwave power $(100 \mathrm{~W}, 2.45 \mathrm{GHz})$ is applied for $30 \mathrm{~s}$; reflected power is measured and simulated and presented in the ranges on the scale from 0 to $100 \mathrm{~W}(\mathrm{~b})$. 

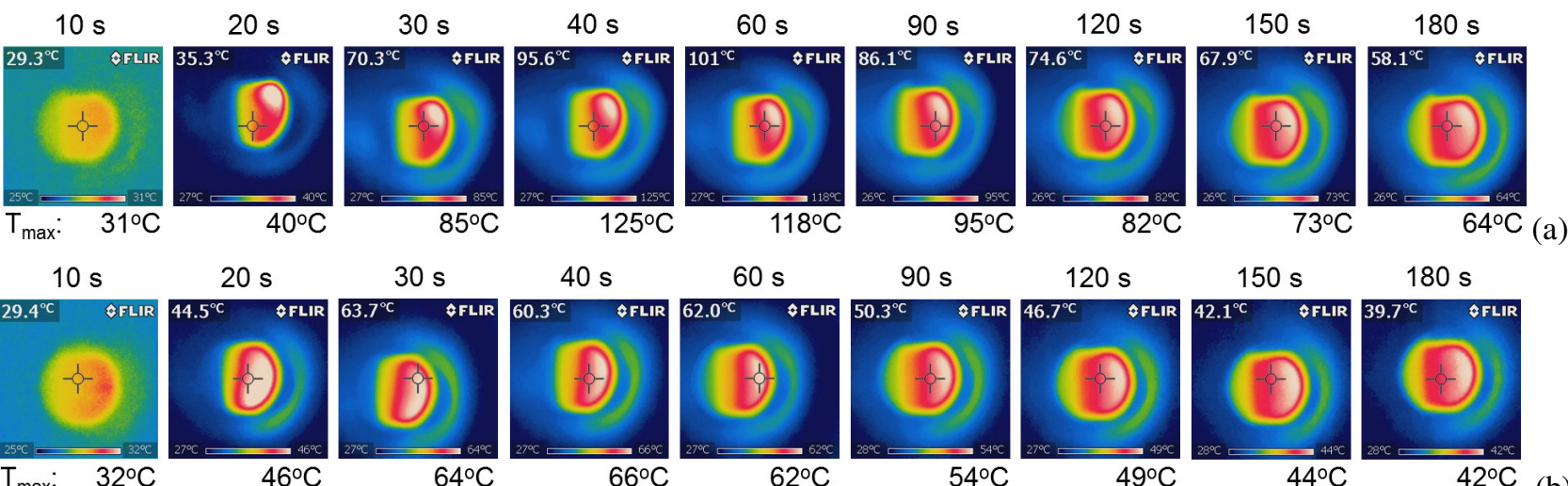

$180 \mathrm{~s}$

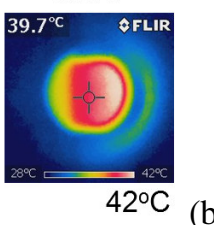

(b)

Fig. 10. Instantaneous (non-normalized) thermal images of the central section of the sample of graphite powder as seen through the side hole of the MiniFlow's waveguide reactor (Fig. 4 (a), (d)) when heating the sample $(w=20 \mathrm{~mm})$ by $100 \mathrm{~W}$ of microwave power; heating time $30 \mathrm{~s}, \mathrm{~s}=85 \mathrm{~mm}$ (a) and $100 \mathrm{~mm}$ (b).

waveguide reactor) and the simulated (for a similar scenario) patterns in Fig. 7: temperature distributions in both cases are relatively spread and conditioned by the regime of wave propagation which, in its turn, is controlled by the position of the shorting wall in the reactor's terminal section.

\section{Discussion and conclusion}

The results in the previous sections show that the principal difficulty in modeling microwave heating of graphite power comes from the lack of precision in specification of complex permittivity and thermal conductivity. Both characteristics depend on particle size and density; in the former, the loss factor is very high; thermal conductivity of the solids fraction of the powder is strongly anisotropic. All that make both measurement and computation (via appropriate physical models) of these parameters quite tricky. Yet, despite the intrinsic uncertainty in characterization of $\varepsilon$ and $k$, the proposed model, as evidenced from the comparison of simulated and measured temperature fields (Figs. 7 and 10) and reflected power (Fig. 9 (b)), appears to be capable of computing evolution of microwave-induced temperature fields with a reasonable accuracy.

Both the loss factor and thermal conductivity are seen to be critical parameters in the formation of a relatively uniform temperature field: if the heating rate is not very high (e.g., due to an elevated level of reflections from the reactor), heat diffusion makes a strong impact on the temperature fields by spreading the heat from the hot spots to the areas with lower temperature. These features are expected to impact the 
characteristics of different microwave heating scenarios with graphite powder and should be carefully addressed in future works.

Since it is technically challenging to experimentally study microwave-induced temperature fields inside graphite powder (as well as other products containing graphite and its derivatives), sufficiently accurate computer models monitoring 3D temperature fields in the course of virtual experimentation appear to be very attractive. The value of such a modeling tool would be even higher for larger samples in larger reactors operating, in contrast to the considered MiniFlow reactors, on their higher modes with multiple minima/maxima of the electric field. The electromagnetic modeling technique used in the present study is proved to be $(i)$ fully capable of accurately handling high modes of arbitrarily-shaped closed microwave systems and (ii) advantageous in solving in multiphysics modeling in microwave power engineering [32]-[34]. However, as seen from the results of this paper, for more precise computations, the electromagnetic-thermal model should also be coupled with a solver accounting for exothermic chemical reactions (in this case, oxidation) as a source of an additional heat.

Our results also demonstrate that, due to the high values of the loss factor and thermal conductivity, microwave-induced temperature distributions in graphite powder may be controlled by the levels of reflections from the reactor that may make a strong impact on the heating rate and the level of absorbed microwave power. Systems fed by solid-state generators and capable of precise tuning their resonances at the operating frequency (like the MiniFlow waveguide reactor with a sliding shorting wall) appear to be particularly convenient for this type of control.

\section{Acknowledgment}

This work was partially supported by an appointment to the Faculty Research Participation Program at the U.S. Air Force Research Laboratory administered by the Oak Ridge Institute for Science and Education (ORISE) through an interagency agreement between the U.S. Department of Energy and U.S. AFRL. It was also partially supported by the Office of the WPI's Dean of Arts and Sciences. The authors are grateful to SAIREM SAS, Neyron, France, for providing the MiniFlow 200SS system for modeling and experimentation, and to Mehulkumar Patel, Huixin He, Germano Iannochione, and Michael Timko for their interest and inspiring discussions.

\section{References}

[1] A. K. Geim, Graphene: status and prospect, Science 324 (2009) 1530-1534. 
[2] V. Singh, D. Joung, L. Zhai, S. Das, S.I. Khondaker, S. Seal, Graphene based materials: past, present and future, Progress in Materials Science 56 (2011) 1178-1271.

[3] C. Soldano, A. Mahmood, E. Dujardin, Production, properties and potential of graphene, Carbon 48 (2010) 2127-2150.

[4] S. Park, R.S. Ruoff, Chemical methods for the production of graphenes, Nature Nanotechnology 4 (2009) 217-224.

[5] Y. Gogotsi, Controlling graphene properties through chemistry, J. Phys. Chem. Lett. 2 (19) (2011) 2509-2510.

[6] O.-Y. Kwon, S.-W. Choi, K.-W. Park, Y.-B. Kwon, The preparation of exfoliated graphite by using microwave, J. Ind. Eng. Chem. 9 (2003) 743-747.

[7] L.M. Viculis, J.J. Mack, O.M. Mayer, H.T. Hahn, R.B. Kaner, Intercalation and exfoliation routes to graphite nanoplatelets, J. Mater. Chem. 15 (2005) 974-978.

[8] B. Tryba, A.W. Morawski, M. Inagaki, Preparation of exfoliated graphite by microwave irradiation, Carbon 43 (2005) 2417-2419.

[9] E.H.L. Falcao, R.G. Blair, J.J. Mack, L.M. Viculis, C.-W. Kwon, M. Bendikov, R.B. Kaner, B.S. Dunn, F. Wudl, Microwave exfoliation of a graphite intercalation compound, Carbon 45 (2007) 13671369.

[10] T. Wei, Z. Fan, G. Luo, C. Zheng, D. Xie, A rapid and efficient method to prepare exfoliated graphite by microwave irradiation, Carbon 47 (2008) 337-339.

[11] G. Xin, W. Hwang, N. Kim, S.M. Cho, H. Chae, A graphene sheet exfoliated with microwave irradiation and interlinked by carbon nanotubes for high-performance transparent flexible electrodes, Nanotechnology 21 (2010) 405201.

[12] Y. Zhu, S. Murali, M.D. Stoller, A. Velamakanni, R.D. Piner, R.S. Ruoff, Microwave assisted exfoliation and reduction of graphite oxide for ultracapacitors, Carbon 48 (7) (2010) 2118-2122.

[13] V. Sridhar, J.-H. Jeon, I.-K. Oh, Synthesis of graphene nano-sheets using eco-friendly chemicals and microwave radiation, Carbon 48 (2010) 2953-2957.

[14] Z. Li, Y. Yao, Z. Lin, K.-S. Moon, W. Lin, C. Wong, Ultrafast, dry microwave synthesis of graphene sheets, J. Mater. Chem. 20 (2010) 4781-4783.

[15] V. Sridhar, J.-H. Jeon, I.-K. Oh, Microwave extraction of graphene from carbon fibers, Carbon 49 (2011) 222-226.

[16] J. Vivas-Castro, G. Rueda-Morales, G. Ortega-Cervantez, L. Moreno-Ruiz, M. Ortega-Aviles, J. Ortiz-Lopez, Synthesis of carbon nanostructures by microwave irradiation, In: S. Yellampalli, (Ed.), Carbon Nanotubes - Synthesis, Characterization, Applications, InTech, 2011, pp. .47-60. 
[17]. H. Hu, Z. Zhao, Q. Zhou, Y. Gogotsi, J. Qiu, The role of microwave absorption on formation of graphene from graphite oxide, Carbon 50 (2012) 3267-3273.

[18] P.L. Chiu, D.D.T. Mastrogiovanni, D. Wei, C. Louis, M. Jeong, G. Yu, P. Saad, C.R. Flach, R.

Mendelsohn, E. Garfunkel, H. He, Microwave- and nitronium ion-enabled rapid and direct production of highly conductive low-oxygen graphene, J. Am. Chem. Soc. 134 (2012) 5850-5856.

[19] U.O. Mendez, O.V. Kharissova, M. Rodriguesz, Synthesis and morphology of nanostructures via microwave heating, Rev. Adv. Mater. Sci. 5 (2003) 398-402.

[20] J.A. Menendez, A. Arenillas, B. Fidalgo, Y. Fernandez, L. Zubizarreta, E.G. Calvo, J.M. Bermudez, Microwave heating processes involving carbon materials, Fuel Processing Technology 91 (2010) 1-8.

[21] S. Chandrasekaran, T. Basak, R. Srivasan, Microwave heating characteristics of graphite based powder mixtures, Intern. Commun. Heat and Mass Transfer 48 (2013) 23-27.

[22] A. Dominguez, J.A. Menendez, M. Inguanzo, J.J. Pis, Investigations into the characteristics of oils produced from microwave pyrolysis of sewage sludge, Fuel Process. Technol. 86 (9) (2005) 1007-1020.

[23] B. Dutta, S.R.S. Dev, V.G.S. Raghavan, Finite element modeling of selective heating in microwave pyrolysis of lignocellulosic biomass, Progress in Electromag. Res. B 56 (2013) 1-24.

[24] N. Yoshikawa, E. Ishizuka, K.-I. Mashiko, S. Taniguchi, Difference in carbo-thermal reduction reaction kinetics of $\mathrm{NiO}$ in microwave E- and H-fields, Mat. Letters 6 (2007) 2096-2099.

[25] K. Kashimura, S. Suzuki, M. Hayashi, T. Mitani, N. Shinohara, K. Nagata, Surface-plasmon-like modes of graphite powder compact in microwave heating, J. App. Phys. 112 (2012) 034905.

[26] J. Zheng, H.-T. Liu, B. Wu, C.-A. Di, Y.-L. Guo, T. Wu, G. Yu, Y.-Q. Liu, D.-B. Zhu, Production of graphite chloride and bromide using microwave sparks, Sci. Reports, 2 (2012) 10.1038/srep00662.

[27] A. Bansal, A.K. Sharma, 3D electromagnetic field simulation of silicon carbide and graphite plate in microwave oven, Int. J. Eng. \& Rob. Res. 1 (1) (2014) 7-12.

[28] M. Hotta, M. Hayashi, M.T. Lanagan, D.K. Agrawal, K. Nagata, Complex permittivity of graphite, carbon black and coal powders in the ranges of X-band frequencies ( 8.2 to $12.4 \mathrm{GHz})$ and between 1 and $10 \mathrm{GHz}$, ISIJ Intern. 51 (11) (2011) 1766-1772.

[29] T.V.C.T. Chan, H.C. Reader, Understanding microwave heating cavities, Artech House, Boston, 2000.

[30] P. Kopyt, M. Celuch-Marcysiak, W.K. Gwarek, Microwave processing of temperature-dependent and rotating objects: development and experimental verification of FDTD algorithms, In: D.C. Folz, J.H. Booske, D.E. Clark, J.F. Gerling (Eds.), Microwave and Radio Frequency Applications, The American Ceramic Society, 2003, pp. 7-16.

[31] M. Soltysiak, M. Celuch, U. Erle, Measured and simulated frequency spectra of the household microwave oven, In 2011 IEEE MTT-S Int. Microwave Symp., Baltimore, MD, June 2011, 978-161284-757-3/11/. 
[32] P. Kopyt, M. Celuch, Coupled electromagnetic-thermodynamic simulations of microwave heating problems using the FDTD algorithm, J. Microwave Power \& Electromag. Energy 41 (4) (2007) 18-29.

[33] P. Kopyt, M. Celuch, Modeling microwave heating in foods, In: M.W. Lorence, P.S. Pesheck (Eds.), Development of Packaging and Products for Use in Microwave Ovens, Woodhead Publishing Ltd., 2009, pp. 250-294.

[34] T.V. Koutchma, V.V. Yakovlev, Computer modeling of microwave heating processes for food preservation, In: M.M. Farid (Ed.), In: Mathematical Analysis of Food Processing, CRC Press, 2010, pp. 625-657.

[35] QuickWave-3D ${ }^{\mathrm{TM}}$, QWED Sp. z o. o., http://www.qwed.com.pl/.

[36] M. Soltysiak, M. Celuch, Influence of dielectric properties on the accuracy of the simulation of microwave heating, In: Proc. 13th Seminar "Computer Modeling in Microwave Engineering \& Applications - Advances in Determining Material Parameters", Thun, Switzerland, 2011, pp. 47-52.

[37] J.-M. Catala-Civera, G. Ianacchione, E.M. Moon, V.V. Yakovlev, Complex permittivity and thermal conductivity of graphite powder - physical models and measurements, J. Microwave Power \& Electromag. Energy 2014 (in press).

[38] A.V. Brovko, E.K. Murphy, V.V. Yakovlev, An artificial neural network technique for determining the volume fraction of solids in particulate materials, In: Proc. 48th IMPI's Microwave Power Symp., New Orleans, LA, 2014, pp. 78-81.

[39] J. E. Atwater, R.R. Wheeler, Jr., Temperature dependent complex permittivities of graphitized carbon blacks at microwave frequencies between 0.2 and 26 GHz, J. Mat. Sci. 39 (2004) 151-157.

[40] Y.C. Li, R.K.Y. Li, S.C. Tjong, Frequency and temperature dependences of dielectric dispersion and electrical properties of polyvinylidene fluoride/expanded graphite composites, J. of Nanomaterials 2010 (2010), ID 261748, 10 p.

[41] A.T.D. Butland, R.J. Maddison, The specific heat of graphite: an evaluation of measurements, J. of Nuclear Materials 49 (1) (1973) 45-56.

[42] W.L. Roberts, T.J. Campbell, G.R. Rapp, Jr., Encyclopedia of Minerals, 2nd Ed., Van Nostrand Reinhold, 1990.

[43] J.W. Zondlo, Graphite: structure, properties, and applications, In: P. Mukhopadhyay, R.K. Gupta (Eds.), Graphite, Graphene, and Their Polymer Nanocomposites, CRC Press, 2012, pp. 1-58.

[44] C.Y. Ho, R.W. Powell, P.E. Liley, Thermal conductivity of the elements: a comprehensive review, J. Phys. and Chem. Ref. Data 3 (suppl. 1) (1974) 150.

[45] S. Zhou, S. Chiang, J. Xu, H. Du, B. Li, C. Xu, F. Kang, Modeling the in-plane thermal conductivity of a graphite/polymer composite sheet with a very high content of natural flake graphite, Carbon 50 (2012) 5052-5061. 
[46] C.T. Hsu, P. Cheng, K.W. Wong, Modified Zehner-Schlunder models for stagnant thermal conductivity of porous media, Int. J. Heat Mass Transfer 37 (17) (1994) 2751- 2759.

[47] M. Kandula, On the effective thermal conductivity of porous packed beds with uniform spherical particles, J. of Porous Media 14 (10) (2011) 919-926.

[48] P. Zehner, E.U. Schlunder, Thermal conductivity of granular materials at moderate temperatures, Chemie. Ingr.-Tech. 42 (1970) 933-941.

[49] W.M. Haynes (Ed), CRC Handbook of Chemistry and Physics, 92nd ed., CRC Press, Boca Raton, 2011.

[50] E.A. Gulbransen, K.F. Andrew, F.A. Brassart, The oxidation of graphite at temperatures of 600 to $1500^{\circ} \mathrm{C}$ and at pressures of 2 to 76 torr of oxygen, J. Electrochemical Society 100 (6) (1963) 476-483. 